\title{
THE AESTHETIC TRIANGLE IN THE ARRANGEMENT OF TEETH: FACE FORM, TOOTH FORM, AND ALIGN. MENT FORM, HARMONIOUS OR GROTESQUE
}

\author{
By A. ALFRED NELSON, D.D.S., Detroit, Michigan
}

(Read before the National Society of Denture Prosthetists, and before The National Dental Association, Milwaukee, Wisconsin, August, 1921)

$I^{x}$ N A PAPER read before the Maryland State Dental Association at Baltimore in 1916, a prominent orthodontist takes issue with the traditional expression that "the human arch varies according to type." He goes to some length to prove that there is no distinct racial type of arch, yet concludes that "environment has produced a distinct American type" and it is this statement that has prompted this work.

In one chapter of his paper he quotes an answer to a letter he wrote to a well-known author and editor relative to determining the shape of the normal arch for a given type and the answer he received is of interest to us as prosthetists. The quotation is as follows: "In reply to your question as to how one is to determine the appropriate type of the arch for a given case, I can only say that you must take into consideration the shape of the head and face in order to determine the shape of the dental arch." As I see the proposition and its bearing upon prosthetic restorations, it is apparent that there is some misunderstanding as to the terms used. I believe the writer has reference to the alignment form of the teeth rather than the arch form. Arch form and alignment form have been greatly intermingled, so that one-half of the profession speak of one and have reference to the other. Prosthetically speaking, the dental arch is that portion of the mouth covered by a denture. Alignment form has reference to the arrangement of the teeth in on or around the dental ridge. I do not believe it is possible for anyone to arbitrarily fix the shape of the dental arch, because nature has done that for us already. The only thing that we can do is to alter the shape slightly when it presents abnormalities, thru the medium of surgical interference or orthodontia.

John Vanderpool, a well-known authority on art, says: "Rules have been laid down by which an ideal standard has been sought to be fixed, the deviation produced by age and sex being taken into account; and while such standards are more or less artificial and not to be slavishly followed to the extent of producing an unnatural uniformity, they certainly are invaluable as expressing a mean which cannot be deviated from to more than a limited extent without transgressing the laws of nature and producing deformity."

Several well-known authorities in art state that the anterior teeth are arranged in the arc of a circle. It is assumed in our profession that the teeth are arranged in the form of a parabolic curve. Lischer so describes them and Ottolengui also' mentions this fact. Stanton claims that each arch possesses 
a particular curve of its own and varies with the individual. However, I have found that there is a distinct relationship between face form, tooth form, arch form and alignment form in nature, and in edentulous patients I have noted that there is a distinct relationship between face form and arch form, especially as related to the upper arch. This being true, it necessarily have been able to distinguish three classes of arches and alignment, the same as faces can be classified. They are the square, the tapering, and the ovoid.

In the square arch the palate is usually broad and shallow and the mandibular ridge is broad in the anterior portion, with a very slight curve from cuspid to cuspid (Fig. 1).

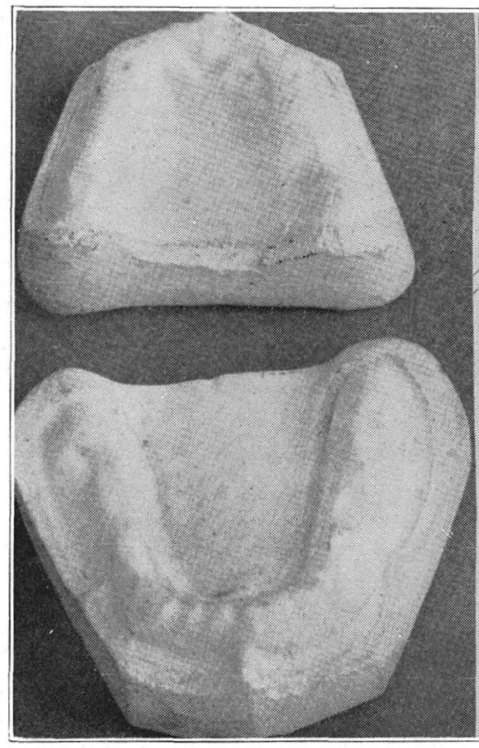

1

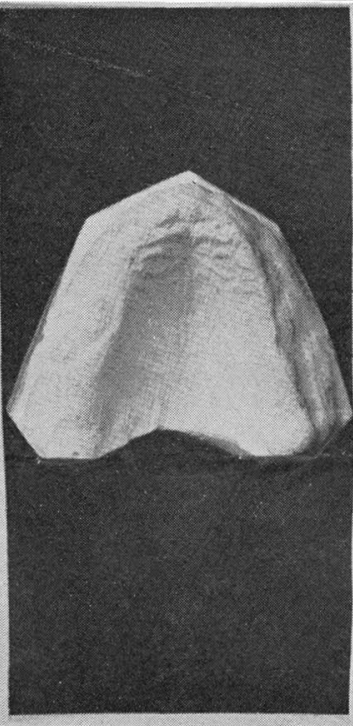

2

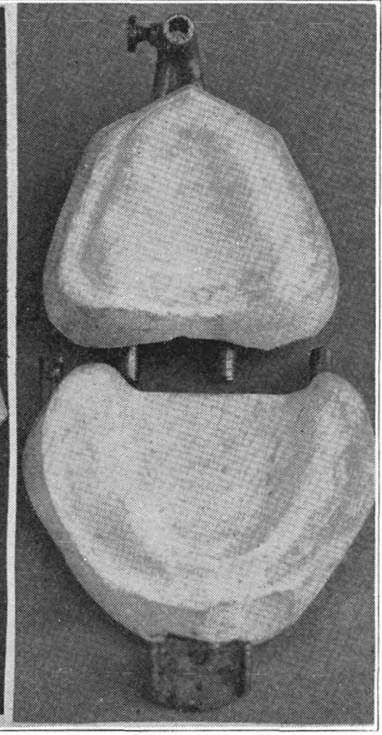

3

Figs. 1-3.-Fig. 1, the square arch. Note the decided $U$ shape of the mandibular ridge, and the broad, shallow palate of the maxilla. Fig. 2, typical tapering upper arch. Due to the limitations of photography it is difficult to show the inverted V-shape of the palate. Fig. 3, tapering arch. Note decided narrowness of mandibular ridge and the lack of tuberosities in the maxilla.

follows that to produce harmony in edentulous cases, we must have the same relationship among all four that we have in nature; that is to say, we must use the proper tooth form and arrange the teeth in the proper alignment to produce this degree of harmony.

From observation and close study of this subject for the past five years, I
In the tapering arch the palate is usually high and inverted V-shaped. The mandibular ridge is very narrow in the anterior portion and presents a decided V-shape (Figs. 2 and 3 ).

In the ovoid arch, the palate is medium high and rounded in the anterior portion of the vault. The mandibular ridge presents a graceful, 


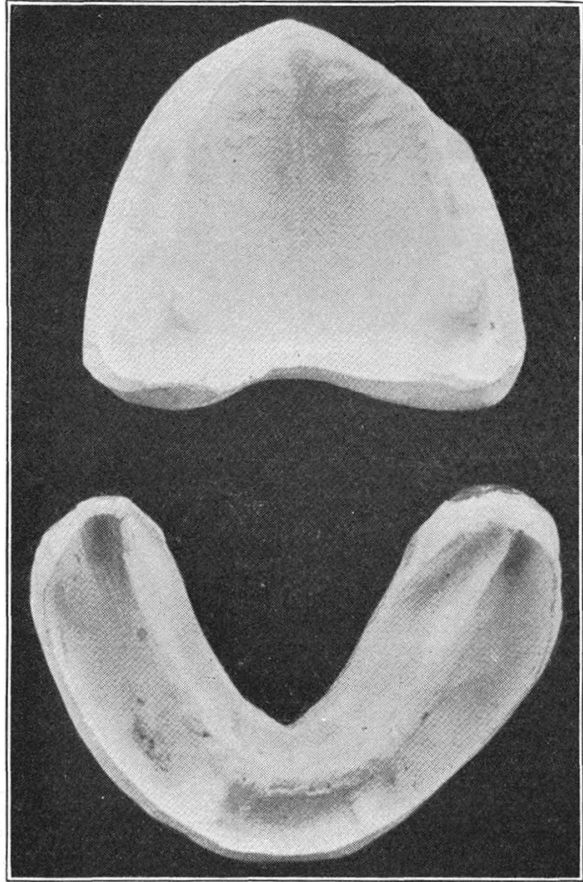

Fig. 4.-Ovoid arch. Note the medium high palate and the rounding of the anterior portion of the vault.

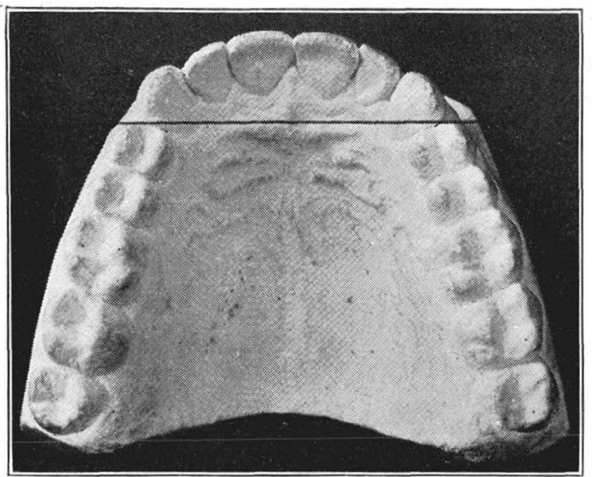

Fig. 5.-The square type of alignment of the anterior teeth. Note the broad, shallow palate and the fact that the arc of the anterior alignment (from cuspid to cuspid) is that of a very large circle. This type of alignment is associated with a square tooth, square face and square arch. (Illustration by courtesy of P. C. Lowery). narrow curve from cuspid eminence to cuspid eminence (Fig. 4).

In the alignment of the teeth, please bear in mind that the chief difference takes place anterior of the first bicuspid and the reference is made only to the maxillae, as the teeth of the mandible follow the general outline of the uppers.

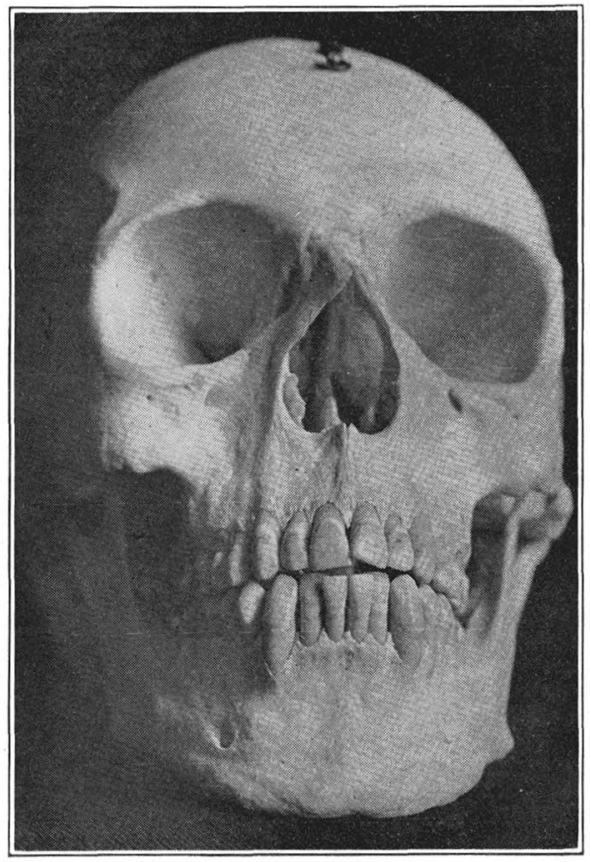

Fig. 6.-This skull is used to show the type of teeth found in the alignment pictured in Fig. 7. The teeth are of the tapering type and the skull that of a person who in normal health presented a tapering face.

We are not concerned with the bicuspids or molars for the reason that the posterior teeth must set over the ridge.

In the square type the alignment of the upper teeth from cuspid to cuspid is in the segment of a large circle, approaching more nearly a straight linc than either of the other types (Fig. 5).

From cuspid to cuspid, the align- 
ment of the six upper anterior teeth in the tapering type is $\mathrm{V}$-shaped with the incisal edges of the teeth well forward

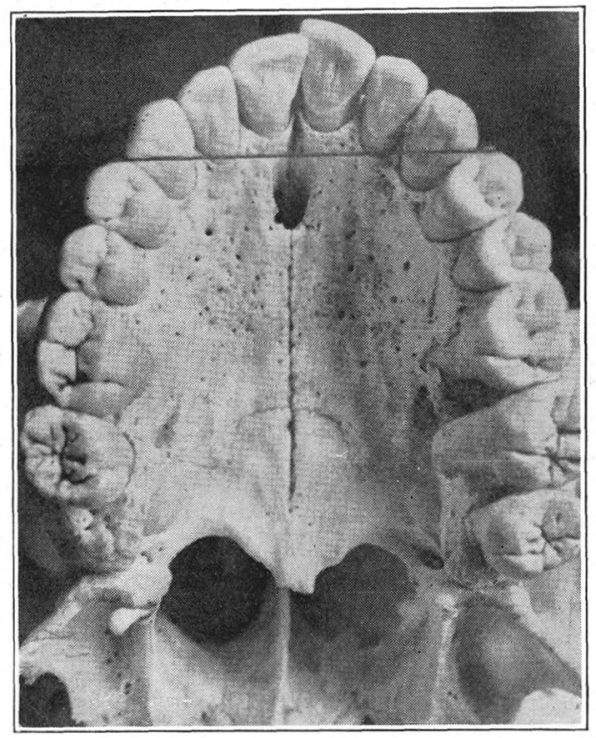

Fig. 7.-The tapering type of alignment of the anterior teeth. This is the alignment view of the case pictured in Fig. 6. Note the decided V-shaped arrangement of the teeth from cuspid to cuspid and the characteristic overlapping of one central on the other.

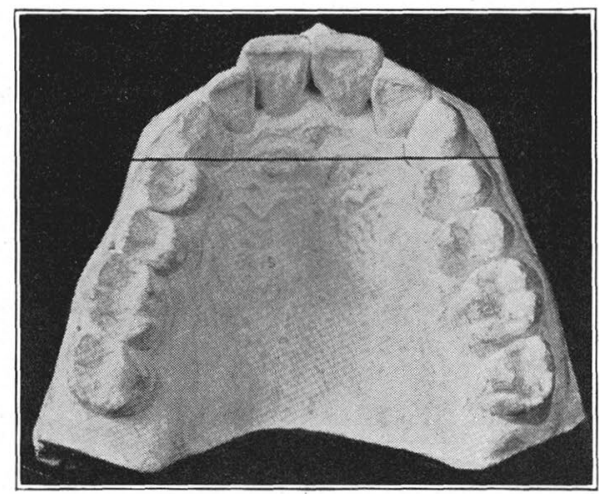

Fig. 8.-A blended alignment of the six anterior teeth. This is a blend between the ovoid and tapering. (Illustration by courtesy of P. C. Lowery). of the cervical. It has been noted as a characteristic of this type that the centrals overlap one another (Figs. 6 and 7 ).

In the ovoid type, the arrangement of the six upper anterior teeth from cuspid to cuspid is in the segment of a small circle, smaller than that of the square. It has been noted as a characteristic of this type that the laterals overlap the centrals slightly at the incisal, but are slightly depressed at the gingival, leaving the mesial shoulder of the cuspid prominent. The centrals are

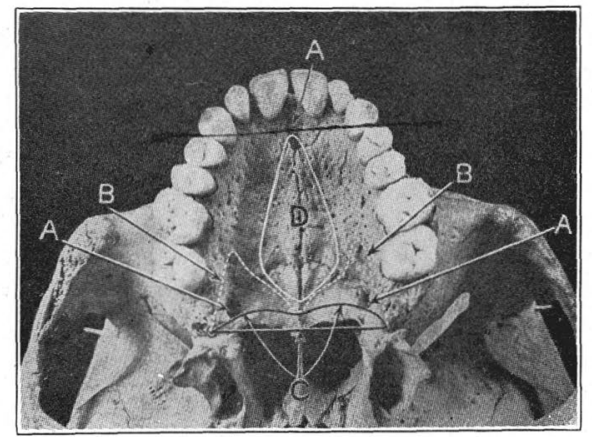

Fig. 9.-The ovoid alignment of the anterior teeth. The arc of the alignment from cuspid to cuspid is that of a very small circle. (Illustration by courtsey of George W. Clapp, Professional Denture Service, Vol. 1).

prominent at the gingival and in a majority of cases there is a space between the two centrals (Figs. 8 and 9).

The square arch and alignment are found in the square type of face; the tapering arch and alignment, in the tapering face, and the ovoid arch and alignment in the ovoid face. I am referring to the pure types. There are blends of these types and especially is this true of the alignment form. In this regard I have noticed three distinct types and three blends of these geometric outlines. The blends are the square 
with the tapering, the tapering with the ovoid and the ovoid with the square (Fig. 10). I do not wish to force the conclusion that the arrangement of teeth should be stereotyped into these alignments. I merely wish to present basic outlines frcm which we may start our composition. 'The deviations are what constitute individuality, just as the portrait artist blocks in all heads alike, using the Greek ideal as a basis from which to start.

Some men contend that there should
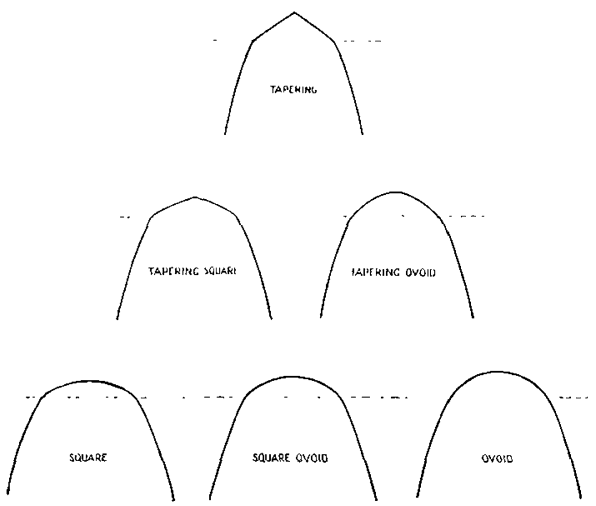

Fig. 10.-_"The aesthetic alignment triangle." "These geometric outlines are intended as basic guides in the arrangement and alignment of the teeth from the optical illusionary and acsthetic standpoint.

be other alignment forms, as for example, the blending of the square and the ovoid in which the square would be dominant and the same blend of type in which the ovoid would be dominant. 'This, to my mind, is nothing more or less than a slight deviation from the pure blend and in those cases to accentuate this domination of a certain type I would use a dominant type of tooth with the blend. For example, in the square-ovoid blend of face in which the square type is dominant, I would select a tooth from class one with the ovoid blend and would begin arrangement from cuspid to cuspid along the pure alignment blend, working in such deviations as I desire, to produce individuality.

It seems to me, and I am not alone in this contention, that there are too many modifications of the pure type of tooth as well as too many sizes of these modi-
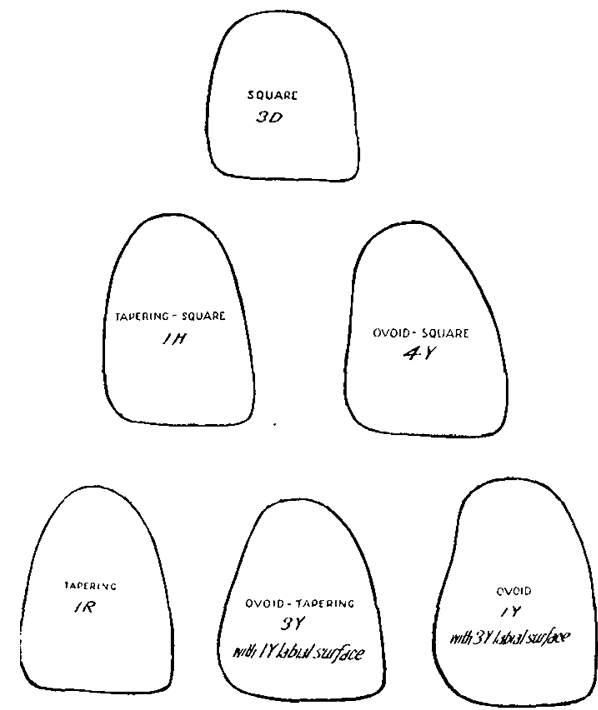

ब⿶at

Fig. 11.-Forms of teeth that will mect all requirements and the mold number of the nearest form in the Trubyte system that approximates these outlines.

fications. I believe that we could limit the forms to six and have three graded sizes of these forms (see Fig. 11), for cxample, small, medium and large.

The definition of "dental aesthetics" as formulated by the Committee on Aesthetics and Art of our society, is as follows: "The science which deduces from nature the rules and principles of facial and dental art." This being so, it is not amiss in a paper of this character to inject a few considerations 
relative to the effects of dentures upon facial expression.

With artificial dentures, it is possible to change a little more than one-third the lower portion of the face; that portion that lies between the bridge of the nose and chin and especially that portion bounded by two diverging lines arising at the lower border of the bridge of the nose and curving downward to in- ponent segments, one with the other, or of the segments with the permanent area, is produced which characterize different physiognomies.

In the preliminary examination of the face from an aesthetic standpoint with a view of harmonizing the features, by supplying the correct "resisting tension" to the soft tissues with the aid of dentures, there are certain prominent fea-

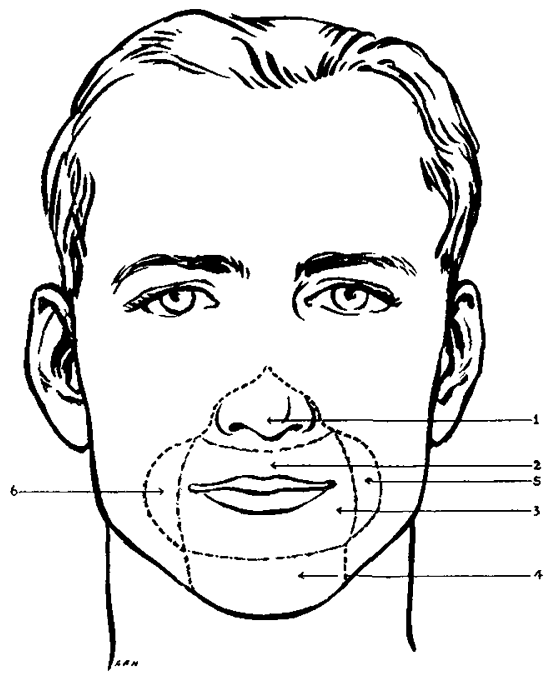

12

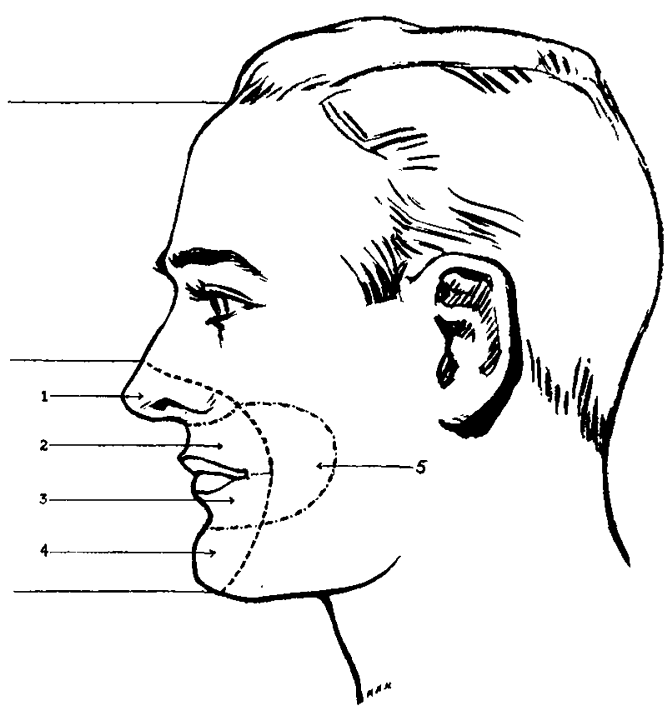

13

Figs. 12 and 13.-Fig. 12, portions of the facc that can be changed with artificial dentures. Fig. 13, side view of Fig. 12.

clude the alae and depressions on either side, portions of each cheek and including the entire chin (see Figs. 12 and 13 ). This area includes the main features of expression and any change of contour within this area will produce an effect upon the entire physiognomy and give a different expression to the countenance. The features outside this area are known as "permanent area" while that included within the boundry is known as "variable." It is in this variable area that lack of harmony among its com- tures to be observed and their relative position noted in both the permanent and variable area.

The variable area is divided into six segments as follows (see Fig. 12): (1) the tip and wings of the nose, including the naso-labial depressions and the upper portion of the upper lip; (2) the lower portion of the upper lip or what is known as cupid's bow; (3) the lower lip; (4) the chin in its entirity; (5) that portion of the cheek lying over the bicuspids and molars on 
the right side; (6) that portion of the cheek lying over the bicuspids and molars on the left side. These six subdivisions are changeable in their relation to one another and also in their individual relation to the features within the permanent area. It is possible to change a portion of the upper lip, the tip and wings of the nose without changing cupid's bow or the lower portion of the upper lip and likewise the reverse is true.
The appearance of its malposition is due largely to retrusion or protrusion of the soft tissues of the variable area.

In other words, we often err in imagining that the relation of the mandible to maxilla in central occlusion is either too open or too closed, because the chin is not in harmonious relation to segments Nos. 1, 2, and 3 when these tissues in all probability are overstrained or lack sufficient resistant tension due to

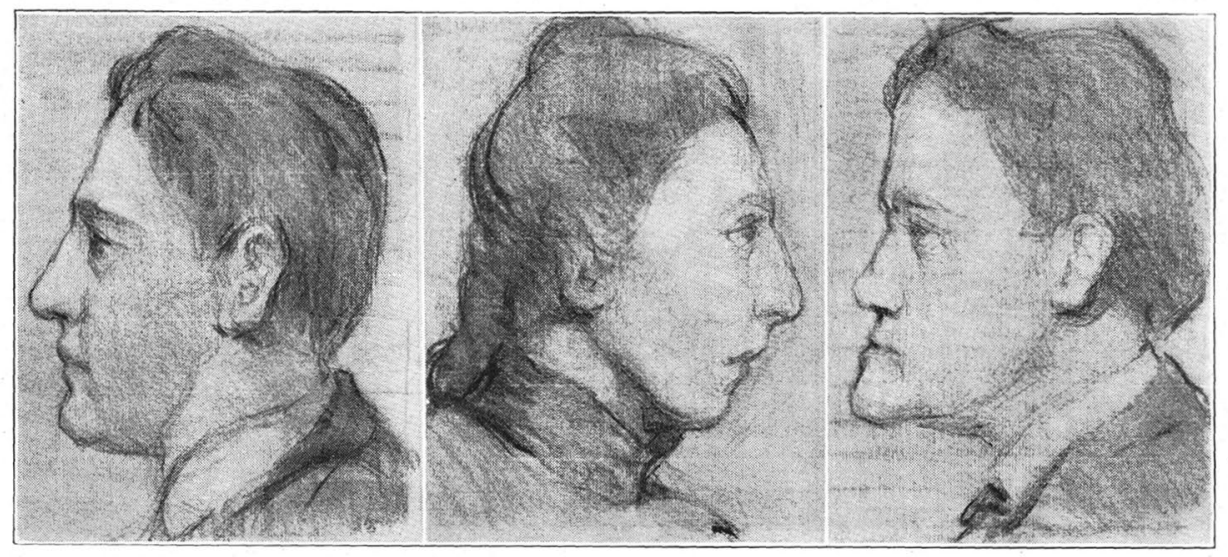

14

15

16

Figs. 14-16.-Fig. 14, the straight profile; Fig. 15, the convex profile; Fig. 16, the concave profile.

In the study of the profiles, a lack of perfect harmony is frequently noted in the position of the chin. The lower jaw appears protruded or retruded with the result that either opening or closing the distance between the arches in central occlusion is often resorted to when in a large percentage of cases the mandible assumes its correct position and the chin its correct relationship to the facioperpendicular line as nature intended. This is due to the involuntary action of the ligaments and the natural relation of the condyle to the glenoid fossae in the relaxed position of the mandible. the occlusion rims inserted for the purpose of securing central occlusion.

There are three general known types of profile: the straight, the convex, and the concave. The straight profile is the Greek ideal face and is the highest ideal of beauty. It has three points of contact with the facio-perpendicular line: the frontal eminence, the middle of the wing of the nose, and the mental eminence (Fig. 14). The convex profile has two points of contact with the facio-perpendicular line: the frontal eminence and the base of the nose. The chin is slightly receding (Fig. 15). The 
concave profile has two points of contact with the facio-perpendicular line: the frontal eminence and the mental eminence. The pure concave is rarely seen, but is often found modified by the straight so that the blend is frequently confused with the true type (Fig. 16).

In securing the relationship of the mandible to the maxilla in central occlusion, it is essential that the operator should bear in mind the types of profile and should be able to recognize the pheral border or the position and inclination of the teeth or the slight building up or depressing of certain contours will often beautify to a remarkable degree the appearance of a face that would otherwise be quite plain and unattractive.

In the normal mouth the orbicularis oris muscle is in a slightly contracted state at all times so that any change in the underlying structure will sooner or later be followed by a similar change in

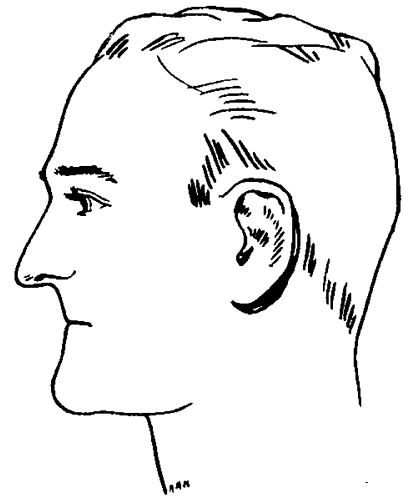

17

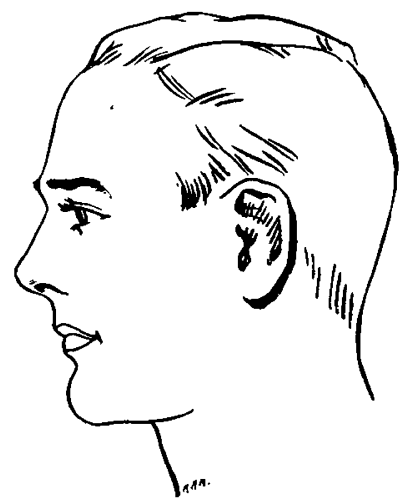

18

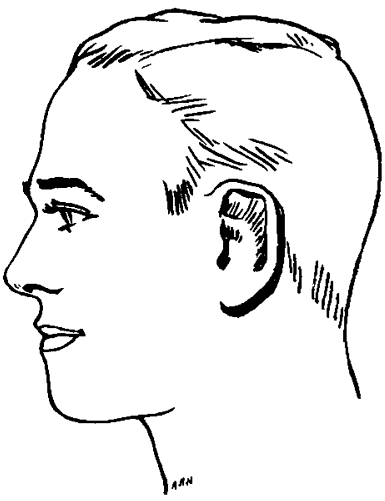

19

Figs. 17-19.-Fig. 17, edentulous case showing contraction of obicularis oris muscle and consequent thinning of the lips; Fig. 18, peripheral border of upper denture too thick; Fig. 19, peripheral border of lower denture too thick.

type to which the patient with whom he is dealing belongs and strive to keep it within its normal class by not having too open or too closed a relation of the jaws to one another.

We should always compare the position of the chin with the stable, permanent features of the face and to the facioperpendicular line of that particular profile. In examining the profile of a patient, the most important thing is the relative position of the chin to the forehead, to the zygomatic arches, and to the bridge of the nose.

It is a noteworthy fact that a very little change in the thickness of the peri- the shape and size of the mouth. This is readily seen in persons wearing full dentures; when the dentures are removed the muscle contracts and the mouth becomes smaller (Fig. 17).

In the try-in of the wax trial dentures, when the upper lip appears protruded, resetting the anterior teeth slightly back or under the ridge and thinning down the labial flanges of the wax denture allows the upper lip to fall into a more graceful and easy pose, leaving the nostrils less broad and open. The upward curve of the nose can be straightened and its pug-like appearance removed (Fig. 18). If the lower lip appears pro- 
truded, the thinning down of the labial flange of the denture and in some cases the shortening of the over-jet and overbite will restore the lower lip to a more pleasing position.

When the protrusion is due to the labial inclination of the teeth with no marked protrusion of the lip or upward curve of the nose, the correction should take place in segment No. 2 and a reduction of this inclination is indicated by straightening the anterior teeth or course the labial flange of the lowers does not interfere with this graceful curve (Figs. 19 and 20).

It often happens that a slight depression of the superior portion of the upper lip with the consequent deepening of naso-labial depressions gives the appearance of protrusion to the lower jaw. If the depression of segment No. 1 be not too pronounced, it may be restored by contouring and building up the cuspid eminence and thickening the periph-

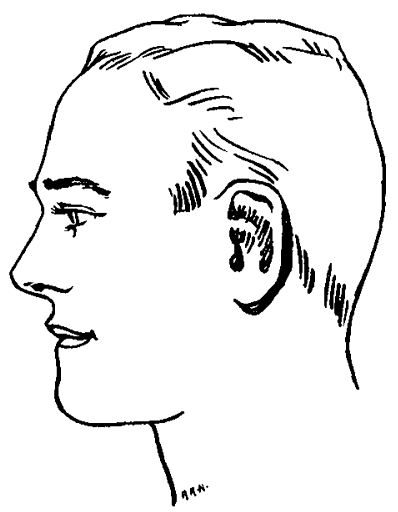

20

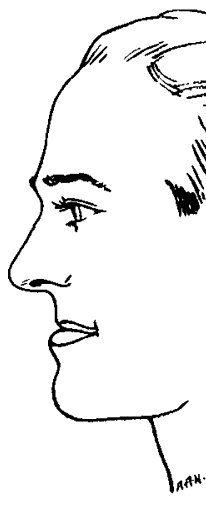

21

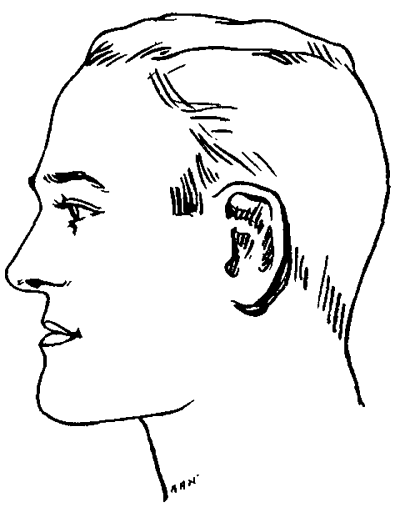

22

Figs. 20-22.-Fig. 20, lower antcriors too far back. Fig. 21, appears at first glance to be a prognathus case but upon close study of the profile it will be found that this is a straight profile. Fig. 22, incisal edges of anterior teeth should be brought out.

setting them back a little farther. In other words, the incisal edge should not be too far forward of the cervical. This applies especially to the upper arch.

There are numerous instances where a pronounced protrusion of the lips with a very unpleasant expression in their management is noted; especially is this true if the occlusion of the anteriors is what may be termed end-on occlusion. The fact that the most natural occluding position of the lower anterior teeth is somewhat posterior to the uppers permits the graceful curve of the lower lip which is so necessary to the aesthetic perfection of the chin, providing of eral margin of the wax trial denture slightly. This facial imperfection is quite often seen in artificial dentures and has given prosthetists quite a little trouble in its management (Figs. 21 and 22).

The position of the bicuspids and molars in their relation to the ridge have a decided influence on segments Nos. 5 and 6 , i. e. that portion of the cheek overlying the bicuspids and molars. If the bicuspids and molars are placed outside, over or inside the ridge, or if the inclination of the long axis of the teeth points in either of these directions, the contour of the cheeks will be affected 
but not necessarily the anterior or labial area. Setting the laterals and cuspids in closer or directly under the ridge will invariably result in giving the cheeks a fuller contour, by relieving the tension of the muscular tissues in the anterior portion, allowing them to collapse posteriorly and thus crowd the cheek segments in the second bicuspid and molar regions.

In examining the physiognomy of a patient, the head should be in an upright position and on a line with that of the operator and the face studied from different angles, while in repose and in action. A careful study of the face in different attitudes of expression should be made with a view to determining the relative position of teeth and facial contour. The value of a careful, preliminary facial and profile examination and comparison cannot be overestimated, for it is the only guide to correct aesthetic treatment.

"Ten-poling" the tissues with high buccal and labial flanges is of no material assistance in the vast majority of cases because the resistance tension of the tissues has not been restored in the correct places.

In denture prosthesis we possess the great advantage over the general practitioner as well as the orthodontist in that we can correct aesthetic deformities by the inclination of the teeth and the depression or contouring of our labial and buccal flanges.

That the laws of harmony govern the arrangement of teeth for the individual case as do these same laws govern the selection of teeth to meet the individual requirements of the case to my mind is a foregone conclusion. And as art in itself is real, nevertheless it is governed by very tangible laws which form the foundation upon which to build; this being true, it is therefore our duty to discover just what these laws are so that the application of this knowledge to the arrangement of teeth in orthodontic as well as prosthetic restorations will result in more nearly establishing a harmonious balance among tooth form, arch form, face form, and alignment iorm.

\section{BIBI,TOGRAPHY}

Case, Calvin S. "Development of Aesthetic Facial Contours," American Textbook of Operative Dentistry, 1897.

Lowery, P. C. "Selection of Teeth for Artificial 'Restorations," Jour. Nat. Dental Assoc., July, 1920.

Report of Aesthetic and Art Committee, 1920. (National Society of Denture Prosthetists).

Williams, J. Leon. A Nerv Classification of Natural and Artificial Teeth, 1915.

Williams, Percy N. "Determining the Shape of the Normal Arch," Dental Cosmos, July, 1917.

Wilson, George H. Prosthetic Dentistry, 1914. 